\title{
Skew-Zigzag Algebras
}

\author{
Chad COUTURE
}

Department of Mathematics and Statistics, University of Ottawa, 585 King Edward Ave, Ottawa, ON K1N 6N5, Canada

E-mail:ccout045@uottawa.ca

Received October 02, 2015, in final form June 17, 2016; Published online June 26, 2016

http://dx.doi.org/10.3842/SIGMA.2016.062

\begin{abstract}
We investigate the skew-zigzag algebras introduced by Huerfano and Khovanov. In particular, we relate moduli spaces of such algebras with the cohomology of the corresponding graph.
\end{abstract}

Key words: zigzag algebra; path algebra; Dynkin diagram; moduli space; graph cohomology

2010 Mathematics Subject Classification: 16G20

\section{Introduction}

Zigzag algebras were introduced by Huerfano and Khovanov in [5] in their categorification of the adjoint representation of simply-laced quantum groups. The zigzag algebra $A(\Gamma)$ is a quotient of the path algebra of the double quiver associated to the Dynkin diagram $\Gamma$ of the quantum group in question. The Grothendieck group of the category of $A(\Gamma)$-modules is then naturally identified with the weight lattice of $\mathfrak{g}$. Zigzag algebras have also recently appeared in categorifications of the Heisenberg algebra. See [2] and [7, Remark 6.2(c)].

In addition to their importance in categorification, zigzag algebras have a variety of nice features, as pointed out in [5]. Examples of such features include the following: they have nondegenerate symmetric trace forms and are quadratic algebras (provided that $\Gamma$ has at least 3 vertices); if $\Gamma$ is a finite Dynkin diagram, then $A(\Gamma)$ is of finite type and its indecomposable representations are enumerated by roots of $\mathfrak{g}$; and if $\Gamma$ is bipartite, then the quadratic dual of the $A(\Gamma)$ is the preprojective algebra of $\Gamma$ for a sink-source orientation.

Skew-zigzag algebras, also introduced in [5], are a generalisation of zigzag algebras. They involve coefficients $v_{b, c}^{a}$ for vertices $a, b, c$ such that $a$ is connected to both $b$ and $c$. When all coefficients are equal to one, they recover the zigzag algebras. The goal of the current paper is to investigate some important properties of skew-zigzag algebras. We provide proofs of some results mentioned in [5] without proof, in addition to proving some results that appear to be new.

We begin with a review of the concepts in graph theory necessary for the current paper in Section 2. In Section 3, we recall the definition of the skew-zigzag algebras, find an explicit basis for them (see Proposition 3.7), and prove that they are graded symmetric algebras (see Proposition 3.9). In Section 4, we describe two moduli spaces of skew-zigzag algebras. The first is the moduli space of skew-zigzag algebras up to isomorphism preserving vertices, which we show to be isomorphic to the graph cohomology of $\Gamma$ (see Theorem 4.8), as stated in [5] without proof (see Remark 4.9). The second is the moduli space of skew-zigzag algebras up to arbitrary isomorphism, which we show to be isomorphic to the quotient of the graph cohomology of $\Gamma$ by a natural action of the automorphism group of $\Gamma$ (see Theorem 4.12). We should note here that we consider the moduli space only as a group, and do not consider any geometric structure. Finally, in Section 5, we discuss an alternate definition of skew-zigzag algebras that has appeared in the literature. We show that this alternate definition is more restrictive (see Proposition 5.3). 


\section{Graph theory background}

Recall that a graph $\Gamma$ is a pair $(V, E)$ where $V$ is a finite set and $E$ is a set consisting of two element subsets of $V$. The elements of $V$ are called vertices and the elements of $E$ are called edges. Note that this implies that we consider graphs with no loops or multiple edges. We will often depict graphs as diagrams, with a node for each vertex and curves between nodes $a$ and $b$ if $\{a, b\} \in E$. If there exist subsets $A, B \subseteq V$ such that $A \sqcup B=V$ and each element of $E$ contains one element of $A$ and one element $B$, then $\Gamma$ is said to be bipartite.

A quiver, $\mathcal{Q}$, is a four-tuple $\left(\mathcal{Q}_{0}, \mathcal{Q}_{1}, s, t\right)$ where $\mathcal{Q}_{0}$ and $\mathcal{Q}_{1}$ are both finite sets and $s, t$ are set maps from $\mathcal{Q}_{1}$ to $\mathcal{Q}_{0}$. The elements of $\mathcal{Q}_{0}$ are again called vertices and the elements of $\mathcal{Q}_{1}$ are called directed edges. For each directed edge $f$, we call $s(f)$ and $t(f)$ the source and target of $f$ (respectively). We will often denote a directed edge with source $a$ and target $b$ by $(a \mid b)$. Throughout this paper we will consider quivers with no parallel edges, i.e., no directed edges $f_{1}, f_{2}$ such that $s\left(f_{1}\right)=s\left(f_{2}\right)$ and $t\left(f_{1}\right)=t\left(f_{2}\right)$.

Example 2.1 (a graph and a quiver). The leftmost diagram below represents the graph $(V, E)$ with $V=\{a, b, c, d\}$ and $E=\{\{a, b\},\{b, c\},\{a, c\},\{a, d\}\}$. The rightmost diagram depicts the quiver $\mathcal{Q}=\left(\mathcal{Q}_{0}, \mathcal{Q}_{1}, s, t\right)$ with $\mathcal{Q}_{0}=\{a, b, c, d\}, \mathcal{Q}_{1}=\{(a \mid b),(a \mid d),(b \mid c),(c \mid d),(d \mid b)\}$ and $s, t: \mathcal{Q}_{1} \rightarrow \mathcal{Q}_{0}$ given by

$$
\begin{aligned}
& s((a \mid b))=s((a \mid d))=a, \quad s((b \mid c))=b, \quad s((c \mid d))=c, \quad s((d \mid b))=d, \\
& t((a \mid b))=t((d \mid b))=b, \quad t((a \mid d))=t((c \mid d))=d, \quad t((b \mid c))=c .
\end{aligned}
$$
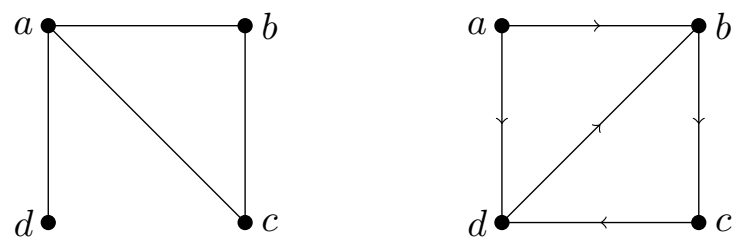

We define a path in a graph $\Gamma$ to be a sequence of vertices $\left(a_{1}, a_{2}, \ldots, a_{n}\right)$ such that $\left\{a_{i}, a_{i+1}\right\} \in$ $E$ for $i=1, \ldots, n-1$. Analogously, we define a path, $P$, in $\mathcal{Q}$ to be a sequence of directed edges $\left(f_{1}, \ldots, f_{n}\right)$ such that the source of $f_{i+1}$ is equal to the target of $f_{i}$ for $1 \leq i \leq n-1$. The source and the target of $P$ are the source of $f_{1}$ and the target of $f_{n}$ (respectively). Furthermore, for any path $P=\left(a_{1}, a_{2}, \ldots, a_{n}\right)$ (respectively $P=\left(f_{1}, \ldots, f_{m}\right)$ ), the length, $\ell(P)$, of $P$ is equal to $n-1$ (respectively $m$ ). We also consider paths of length 0 which start and end at the same vertex $a$, called empty paths or trivial paths and denoted $(a)$. We shall use $\mathcal{Q}_{i}$ to denote the paths of length $i$ in $\mathcal{Q}$. In addition, we shall use $\left(a_{1}\left|a_{2}\right| \ldots \mid a_{n}\right)(n \geq 1)$ to denote a path $\left(f_{1}, \ldots, f_{n-1}\right)$ such that $s\left(f_{i}\right)=a_{i}$ and $t\left(f_{i}\right)=a_{i+1}$ for $1 \leq i \leq n-1$ in $\mathcal{Q}$. We say that $\Gamma$ is connected if for any vertices $a$ and $b$, there exits a path $P$ between $a$ and $b$.

Example 2.2 (paths). In the graph of Example 2.1, $(a, b, c, a, d)$ is a path from $a$ to $d$ of length 4 . In the quiver of Example 2.1, $(a|b| c \mid d)$ is a path of length 3. However, $(a|b| d)$ is not a path in the quiver of Example 2.1.

We define the double graph of $\Gamma$, denoted $D \Gamma$, to be the quiver consisting of the vertices of $\Gamma$ and for each edge $\{a, b\}$ of $\Gamma, D \Gamma$ has two edges $f_{1}$ and $f_{2}$ with $s\left(f_{1}\right)=t\left(f_{2}\right)=a$ and $s\left(f_{2}\right)=t\left(f_{1}\right)=b$.

Example 2.3 (double graph). Let $\Gamma$ be the graph in Example 2.1. Its double graph, $D \Gamma$, is the following quiver. 


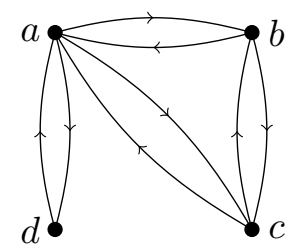

A path $C=\left(a_{1}, a_{2}, \ldots, a_{n}\right)$ in $\Gamma$ is said to be a cycle if $a_{1}=a_{n}$. Then we define $V_{C}$ and $E_{C}$ to be the sets $\left\{a_{i} \mid 1 \leq i \leq n-1\right\}$ and $\left\{\left\{a_{i}, a_{i+1}\right\} \mid 1 \leq i \leq n-1\right\}$ respectively. Similarly, a path $C=\left(a_{1}\left|a_{2}\right| \ldots \mid a_{n}\right)$ in $\mathcal{Q}$ is said to be a cycle if $a_{1}=a_{n}$. Then we define $V_{C}$ and $E_{C}$ to be the sets $\left\{a_{i} \mid 1 \leq i \leq n-1\right\}$ and $\left\{\left\{a_{i}, a_{i+1}\right\} \mid 1 \leq i \leq n-1\right\}$ respectively. For each vertex $a \in V$, we define the degree of $a$, denoted $\operatorname{deg}(a)$, to be the cardinality of the set $\{e \in E \mid a \in e\}$. Furthermore, for any cycle $C$ (in a quiver or a graph), we define the degree in $C$ of a vertex $a \in V_{C}$, denoted $\operatorname{deg}_{C}(a)$, to be the cardinality of the set $\left\{e \in E_{C} \mid a \in e\right\}$. Finally, we say that $C$ is a simple cycle if for all $a \in V_{C}$ we have $\operatorname{deg}_{C}(a)=2$.

We say that a graph $\Gamma^{\prime}=\left(V^{\prime}, E^{\prime}\right)$ is a subgraph of $\Gamma=(V, E)$ if $V^{\prime} \subseteq V$ and $E^{\prime} \subseteq E$. If $T$ is a connected graph that does not contain any simple cycles, then $T$ is said to be a tree. Let $T=\left(V_{T}, E_{T}\right)$ be a subgraph of $\Gamma$. If $V_{T}=V$ and if $T$ is a tree, then $T$ is called a spanning tree of $\Gamma$. Every connected graph has at least one spanning tree (see, for instance, [4, Section 1.5]). If $\Gamma=(V, E)$ is a connected graph and $T=\left(V_{T}, E_{T}\right)$ is a spanning tree of $\Gamma$, then $\left|E_{T}\right|=|V|-1$.

\section{$3 \quad$ Skew-zigzag algebras}

In this section we introduce our main objects of study, the skew-zigzag algebras, and prove some basic facts about them that are stated without proof in the literature. Throughout this section, we fix a field $\mathbb{k}$ and a connected graph $\Gamma=(V, E)$.

\subsection{Definitions}

We first recall the definition of the path algebra of a quiver. We refer the reader to Chapter 2 of [1] for further details. The path algebra of a quiver $\mathcal{Q}$, denoted $\mathbb{k} \mathcal{Q}$, is the vector space with basis consisting of all paths. We define the concatenation of two paths $\left(a_{1}\left|a_{2}\right| \ldots \mid a_{n}\right)$ and $\left(a_{1}^{\prime}\left|a_{2}^{\prime}\right| \ldots \mid a_{m}^{\prime}\right)$ to be the path $\left(a_{1}\left|a_{2}\right| \ldots\left|a_{n}=a_{1}^{\prime}\right| a_{2}^{\prime}|\ldots| a_{m}^{\prime}\right)$ when $a_{n}=a_{1}^{\prime}$ and 0 otherwise. Then, we define the multiplication of two paths $P_{1} * P_{2}$ to be the concatenation of paths. The path algebra is an $\mathbb{N}$-graded algebra, i.e.,

$$
\mathbb{k} \mathcal{Q}=\bigoplus_{i=0}^{\infty} \mathbb{k} \mathcal{Q}_{i}
$$

where each $\mathbb{k} \mathcal{Q}_{i}$ is the $\mathbb{k}$-vector space spanned by all paths of length $i$, and $\mathbb{k} \mathcal{Q}_{i} \mathbb{k} \mathcal{Q}_{j} \subseteq \mathbb{k} \mathcal{Q}_{i+j}$ for all $i, j$. We call an element of $\mathbb{k} \mathcal{Q}$ homogeneous of degree $i$ if it lies in $\mathbb{k} \mathcal{Q}_{i}$. We will be mostly interested in the case where $\mathcal{Q}=D \Gamma$.

Recall that an ideal $I$ of a graded algebra $A=\bigoplus_{i=0}^{\infty} A_{i}$ is called graded or homogeneous if it is generated by homogeneous elements of $A$. Equivalently, $I$ is homogeneous if $I=\bigoplus_{i=0}^{\infty}\left(I \cap A_{i}\right)$.

Definition 3.1 (skew-zigzag coefficients). A set $v=\left(v_{b, c}^{a} \in \mathbb{k} \mid\{a, b\},\{a, c\} \in E\right)$ is a collection of skew-zigzag coefficients for the graph $\Gamma=(V, E)$ if it satisfies the following three conditions:

- $v_{b, b}^{a}=1$ for all $\{a, b\} \in E$,

- $v_{b, c}^{a} v_{c, b}^{a}=1$ for all $\{a, b\},\{a, c\} \in E$, and

- $v_{b, c}^{a} v_{c, d}^{a} v_{d, b}^{a}=1$ for all $\{a, b\},\{a, c\},\{a, d\} \in E$. 
From now on, whenever we say that two vertices are connected, we shall mean that there is an edge between these two vertices. We are now ready to recall the definition of the algebras defined originally in [5, p. 527].

Definition 3.2 (skew-zigzag algebra). Let $v$ be a collection of skew-zigzag coefficients.

- If $\Gamma$ only contains one vertex, then we define $A_{v}(\Gamma)$ to be the algebra generated by 1 and $X$ with $X^{2}=0$.

- If $\Gamma$ contains two vertices, then we define $A_{v}(\Gamma)$ to be the quotient algebra of the path algebra of $D \Gamma$ by the two-sided ideal generated by all paths of length greater than two.

- If $\Gamma$ has at least three vertices, we define $I_{v}$ to be the ideal generated by

a) paths of the form $\left(a_{1}\left|a_{2}\right| a_{3}\right)$ for all $a_{1}, a_{2}, a_{3}$ in $\Gamma$ such that $a_{1}$ is connected to $a_{2}, a_{2}$ is connected to $a_{3}$ and $a_{1} \neq a_{3}$,

b) elements of the form $\left(a_{1}\left|a_{2}\right| a_{1}\right)-v_{a_{2}, a_{3}}^{a_{1}}\left(a_{1}\left|a_{3}\right| a_{1}\right)$ for all $a_{1}, a_{2}, a_{3} \in V$ such that $a_{1}$ is connected to $a_{2}, a_{3}$.

We then define $A_{v}(\Gamma)$ to be the quotient algebra of the path algebra of $D \Gamma$ by the ideal $I_{v}$.

We call $A_{v}(\Gamma)$ the skew-zigzag algebra of $\Gamma$. When $v_{a_{2}, a_{3}}^{a_{1}}=1$ for all vertices $a_{1}, a_{2}, a_{3}$ such that $a_{1}$ is connected to both $a_{2}$ and $a_{3}$, then we call $A_{v}(\Gamma)$ the zigzag algebra of $\Gamma$ and denote it $A(\Gamma)$.

Remark 3.3. Notice that $I_{v}$ is generated by homogeneous elements of $\mathbb{k} \Gamma$ and hence is a graded ideal. Therefore, the skew-zigzag algebra inherits a grading of its own. More precisely, we have

$$
A_{v}(\Gamma)=\bigoplus_{i=0}^{\infty} \mathbb{k} \mathcal{Q}_{i} /\left(I_{v} \cap \mathbb{k} \mathcal{Q}_{i}\right)=\bigoplus_{i=0}^{\infty}\left(\mathbb{k} \mathcal{Q}_{i}+I_{v}\right) / I_{v}
$$

Moreover, if $\Gamma$ contains at least two vertices, $I_{v}$ is generated by elements of $\mathbb{k} \mathcal{Q}_{2}$. Thus $I_{v}$ is contained in $\bigoplus_{i \geq 2} \mathbb{k} \mathcal{Q}_{i}$. Consequently, any path of length less than two cannot sit inside $I_{v}$.

For a path $P$ in $D \Gamma$, we let $[P]$ denote the equivalence class of $P$ in $A_{v}(\Gamma)$. Similarly, we shall use the notation $\left[a_{1}\left|a_{2}\right| \ldots \mid a_{n}\right]$ to denote the equivalence class of $\left(a_{1}\left|a_{2}\right| \ldots \mid a_{n}\right), n \geq 1$, in $A_{v}(\Gamma)$.

\subsection{Bases}

Our next goal is to describe an explicit basis of the skew-zigzag algebra. We begin with a few technical results. Throughout this subsection, we fix a collection $v$ of skew-zigzag coefficients for the connected graph $\Gamma=(V, E)$.

Lemma 3.4. Let $a, a_{1}, \ldots, a_{n} \in V$ with $n \geq 2$, and suppose that $a$ is connected to $a_{1}, \ldots, a_{n}$. Then

$$
v_{a_{1}, a_{2}}^{a} v_{a_{2}, a_{3}}^{a} \cdots v_{a_{n-1}, a_{n}}^{a}=v_{a_{1}, a_{n}}^{a} .
$$

Proof. We shall proceed by induction on $n$. For $n=2$, this trivially holds. Now suppose that (3.1) holds for some integer $n \geq 2$. Let us prove that it holds for $n+1$. We have

$$
\prod_{i=1}^{n} v_{a_{i}, a_{i+1}}^{a}=\left(\prod_{i=1}^{n} v_{a_{i}, a_{i+1}}^{a}\right) v_{a_{n+1}, a_{n-1}}^{a} v_{a_{n-1}, a_{n+1}}^{a}=\left(\prod_{i=1}^{n-2} v_{a_{i}, a_{i+1}}^{a}\right) v_{a_{n-1}, a_{n+1}}^{a}=v_{a_{1}, a_{n+1}}^{a},
$$

where the last equality follows from the induction hypothesis. 
Corollary 3.5. For all $n \geq 2$, we have

$$
v_{a_{1}, a_{2}}^{a} v_{a_{2}, a_{3}}^{a} \cdots v_{a_{n-1}, a_{n}}^{a} v_{a_{n}, a_{1}}^{a}=1 .
$$

Proof. Suppose $n \geq 2$. By Lemma 3.4, we have

$$
v_{a_{1}, a_{2}}^{a} v_{a_{2}, a_{3}}^{a} \cdots v_{a_{n-1}, a_{n}}^{a} v_{a_{n}, a_{1}}^{a}=v_{a_{1}, a_{n}}^{a} v_{a_{n}, a_{1}}^{a}=1 .
$$

Lemma 3.6. Let $P_{1}, \ldots, P_{n}$ be paths in a quiver, no two of which have the same source and target. If $\left[P_{i}\right] \neq 0$ for all $i=0, \ldots, n$ then $\left[P_{1}\right], \ldots,\left[P_{n}\right]$ are linearly independent.

Proof. Suppose there exist $\alpha_{1}, \ldots, \alpha_{n} \in \mathbb{k}$ such that

$$
\alpha_{1}\left[P_{1}\right]+\cdots+\alpha_{n}\left[P_{n}\right]=0 .
$$

Then, for all $i=1, \ldots, n$, we obtain

$$
0=\alpha_{1}\left[s\left(P_{i}\right)\right]\left[P_{1}\right]\left[t\left(P_{i}\right)\right]+\cdots+\alpha_{i}\left[s\left(P_{i}\right)\right]\left[P_{i}\right]\left[t\left(P_{i}\right)\right]+\cdots+\alpha_{n}\left[s\left(P_{i}\right)\right]\left[P_{n}\right][t(P i)]=\alpha_{i}\left[P_{i}\right] .
$$

Hence, we must have $\alpha_{i}=0$ for all $i=1, \ldots, n$. Thus, $\left[P_{1}\right], \ldots,\left[P_{n}\right]$ are linearly independent.

We are now in a position to determine a basis of the skew-zigzag algebra. In particular, this gives us the dimension of the zigzag algebra, which was stated in [5, Section 3] without proof.

Proposition 3.7 (basis of the skew-zigzag algebra). Recall that $\Gamma=(V, E)$ is a connected graph and $v$ is a collection of skew-zigzag coefficients.

- If $\Gamma$ only has one vertex, then $\{1, X\}$ is a basis for $A_{v}(\Gamma)$.

- If $\Gamma$ has two vertices, $a$ and $b$, then $\{[a],[b],[a \mid b],[b \mid a],[a|b| a],[b|a| b]\}$ is a basis for $A_{v}(\Gamma)$.

- If $\Gamma$ has three or more vertices, for all $x \in V$ we define $V_{x}$ to be set of all vertices that are connected to $x$ and we fix a vertex $y_{x} \in V_{x}$. Then

$$
J:=\left\{[a],[b \mid c],\left[x\left|y_{x}\right| x\right] \mid a, x \in V,\{b, c\} \in E\right\}
$$

is a basis for $A_{v}(\Gamma)$. In particular, we have $\operatorname{dim} A_{v}(\Gamma)=2|V|+2|E|$.

Proof. The author would like to thank a referee for bringing into light a much simpler proof of this proposition.

The first two claims are obvious. Therefore, we assume that $\Gamma$ has at least three vertices. Recall that the ideal $I_{v}$ is generated by the set

$$
X_{v}:=\left\{(a|b| c),(x|y| x)-v_{y, z}^{x}(x|z| x) \mid\{a, b\},\{b, c\},\{x, y\},\{x, z\} \in E, a \neq c\right\},
$$

and hence

$$
[a|b| c]=0, \quad[x|y| x]=v_{y, z}^{x}[x|z| x]
$$

for all $\{a, b\},\{b, c\},\{x, y\},\{x, z\} \in E$ with $a \neq c$. Note that the first equality implies that any path with three consecutive pairwise distinct vertices is equivalent to zero. Now, consider a path of the form $(a|b| a \mid b)$ where $\{a, b\} \in E$. Since $\Gamma$ is connected and has at least three vertices, either $a$ or $b$ is connected to a third vertex $d \neq a, b$. Suppose $b$ is connected to $d$. (The case that $a$ is connected to $d$ is analogous.) Then, (3.3) yields

$$
[a|b| a \mid b]=[a \mid b][b|a| b]=v_{a, d}^{b}[a \mid b][b|d| b]=[a|b| d][d \mid b]=0 .
$$


Thus, any path of length 3 or greater has an equivalence class equal to 0 . So $A_{v}(\Gamma)$ only contains elements of degree 0,1 or 2. It is clear that $\{[a] \mid a \in V\}$ and $\{[a \mid b] \mid\{a, b\} \in E\}$ are bases for $\left(A_{v}(\Gamma)\right)_{0}$ and $\left(A_{v}(\Gamma)\right)_{1}$ respectively since $I_{v}$ is concentrated in degrees 2 and higher. Now, let $x \in V$. Notice that for any $a \in V_{x}$, we have $[x|a| x]=v_{a, y_{x}}^{x}\left[x\left|y_{x}\right| x\right]$. So any element in $\left([x] A_{v}(\Gamma)[x]\right)_{2}$ can be written as some nonzero scalar times $\left[x\left|y_{x}\right| x\right]$. Thus, $\left\{\left[x\left|y_{x}\right| x\right] \mid x \in V\right\}$ is a spanning set for $\left(A_{v}(\Gamma)\right)_{2}$ and so, by Lemma 3.6, it is a basis of $\left(A_{v}(\Gamma)\right)_{2}$. Consequently, $J$ is a basis for $A_{v}(\Gamma)$.

Finally, notice that we have

$$
J=\{[a] \mid a \in V\} \sqcup\{[a \mid b] \mid\{a, b\} \in E\} \sqcup\left\{\left[x\left|y_{x}\right| x\right] \mid x \in V\right\} .
$$

Thus, $|J|=|\{[a] \mid a \in V\}|+|\{[a \mid b] \mid\{a, b\} \in E\}|+\left|\left\{\left[x\left|y_{x}\right| x\right] \mid x \in V\right\}\right|=2|V|+2|E|$.

\subsection{Skew-zigzag algebras as Frobenius algebras}

We begin by recalling the concept of a Frobenius algebra, referring the reader to [6] for further details. Let $f$ be a bilinear form

$$
f: V \times V \rightarrow \mathbb{k}
$$

where $V$ is a vector space of finite dimension over the field $\mathbb{k}$. A trace map gives rise to a bilinear form $(x, y) \mapsto \operatorname{tr}(x y)$.

Let $A$ be a $\mathbb{k}$-algebra. Let tr be a $\mathbb{k}$-linear map

$$
\operatorname{tr}: A \rightarrow \mathbb{k}
$$

We call tr a trace map. A trace map gives rise to a bilinear form $(x, y) \mapsto \operatorname{tr}(x y)$.

Definition 3.8 (Frobenius and symmetric algebra). Let $A$ be a finite-dimensional unital associative $\mathbb{k}$-algebra. If there exists a nondegenerate trace map $\operatorname{tr}: A \rightarrow \mathbb{k}$, then $A$ is said to be a Frobenius algebra. Moreover, if there exists a nondegenerate symmetric trace map, then $A$ is said to be a symmetric Frobenius algebra or simply a symmetric algebra.

Recall that $\Gamma=(V, E)$ is a connected graph. Let $v$ be a collection of skew-zigzag coefficients, and let $P$ be a path in $D \Gamma$. Throughout this article, we shall define the source and the target of the equivalence class $[P], s([P])$ and $t([P])$, to be $[s(P)]$ and $[t(P)]$ respectively. If $P_{1}$ and $P_{2}$ are both trivial paths or paths of length 1 then we have $\left[P_{1}\right]=\left[P_{2}\right]$ if and only if $P_{1}=P_{2}$. If $P_{1}$ and $P_{2}$ are paths of length 2 then $\left[P_{1}\right]$ is a scalar multiple of $\left[P_{2}\right]$ if and only if $s\left(P_{1}\right)=s\left(P_{2}\right)$ and $t\left(P_{1}\right)=t\left(P_{2}\right)$. Thus $[s(P)]$ and $[t(P)]$ are well-defined.

If $[P] \neq 0$, we define the length of $[P]$, denoted $\ell([P])$, to be $\ell(P)$. If $[P]=0$, then we simply define $\ell([P])$ to be 0 . Since $I_{v}$ is generated by homogeneous elements of the same degree, $\ell([P])$ is well-defined.

Let $J$ be as in (3.2) and define the $\mathbb{k}$-linear map $\operatorname{tr}: A_{v}(\Gamma) \rightarrow \mathbb{k}$ on the elements of $J$ as follows:

$$
\operatorname{tr}([P])= \begin{cases}1 & \text { if } \ell([P])=2, \\ 0 & \text { otherwise. }\end{cases}
$$

For any path $P=(a|b| a)$, we let $v_{P}=v_{b, y_{a}}^{a}$ where $y_{a}$ is defined as in Proposition 3.7.

Proposition 3.9. Recall that $\Gamma=(V, E)$ is a connected graph, and let $v$ be a collection of skewzigzag coefficients. Then $A_{v}(\Gamma)$ is a graded Frobenius algebra. In addition, $A(\Gamma)$ is a graded symmetric algebra. 
Proof. As noted in [5, Proposition 1], zigzag algebras are symmetric algebras. Furthermore, as stated in [5, Section 4.5], skew-zigzag algebras are Frobenius algebras. This follows from the fact that a basic finite-dimensional algebra over an algebraically closed field is Frobenius if the socle of any projective indecomposable module is simple, and the map $P / \operatorname{rad}(P) \mapsto \operatorname{soc}(P)$ is a bijection onto the set of isomorphism classes of simple modules. The fact that the Frobenius form is as defined above then follows from Propositions 1.10 .18 and 3.6.14 of [9]. It is also possible to prove directly that the trace map defined above has the desired properties.

\section{Moduli spaces of skew-zigzag algebras}

In this section we describe the moduli spaces of skew-zigzag algebras up to various types of isomorphism. We will see that such moduli spaces are related to the cohomology of the corresponding graph. As noted in the introduction, we will only consider the group structure, and not any geometric structure, on the moduli spaces to be introduced below. Throughout this section we fix a field $\mathbb{k}$ that contains square roots.

Let $\Gamma=(V, E)$ be a connected graph and $v$ a collection of skew-zigzag coefficients. Let $P=\left(a_{1}|\ldots| a_{n}\right)$ be a path in $\Gamma$. Define

$$
v_{P}^{\mathrm{path}}=\prod_{i=2}^{n-1} v_{a_{i-1}, a_{i+1}}^{a_{i}} .
$$

We call $v_{P}^{\text {path }}$ the product of $v$ along $P$. If $P$ is a cycle, then we also define

$$
v_{P}^{\text {cycle }}=v_{a_{n-1}, a_{2}}^{a_{1}} \prod_{i=2}^{n-1} v_{a_{i-1}, a_{i+1}}^{a_{i}}=v_{a_{n-1}, a_{2}}^{a_{1}} v_{P}^{\text {path }},
$$

and call $v_{P}^{\text {cycle }}$ the cycle product of $v$ along $P$. Furthermore, we define $P^{*}=\left(a_{n}|\ldots| a_{1}\right)$. It is easily seen that $\left(v_{P}^{\text {path }}\right)^{-1}=v_{P^{*}}^{\text {path }}$.

Let $P_{1}=\left(a_{1}|\ldots| a_{n}\right)$ and $P_{2}=\left(a_{n}|\ldots| a_{m}\right)$ be two paths. Notice that we have

$$
\begin{aligned}
v_{P_{1} P_{2}}^{\text {path }} & =\left(\prod_{i=2}^{m-1} v_{a_{i-1}, a_{i+1}}^{a_{i}}\right)=\left(\prod_{i=2}^{n-1} v_{a_{i-1}, a_{i+1}}^{a_{i}}\right) v_{a_{n-1}, a_{n+1}}^{a_{n}}\left(\prod_{i=n+1}^{m-1} v_{a_{i-1}, a_{i+1}}^{a_{i}}\right) \\
& =v_{P_{1}}^{\text {path }} v_{a_{n-1}, a_{n+1}}^{a_{n}} v_{P_{2}}^{\text {path }} .
\end{aligned}
$$

If, in addition, $P_{1}$ and $P_{2}$ are cycles, then $P_{1} P_{2}$ is also a cycle and thus,

$$
\begin{aligned}
v_{P_{1} P_{2}}^{\text {cycle }} & =v_{a_{m-1}, a_{2}}^{a_{1}} v_{P_{1} P_{2}}^{\text {path }}=v_{a_{m-1}, a_{2}}^{a_{1}} v_{a_{n-1}, a_{n+1}}^{a_{n}} v_{P_{1}}^{\text {path }} v_{P_{2}}^{\text {path }} \\
& =v_{a_{m-1}, a_{n+1}}^{a_{1}} v_{a_{n-1}, a_{2}}^{a_{1}} v_{a_{n-1}, a_{n+1}}^{a_{n}} v_{P_{1}}^{\text {path }} v_{P_{2}}^{\text {path }} \\
& =v_{a_{n-1}, a_{2}}^{a_{1}} v_{a_{m-1}, a_{n+1}}^{a_{n}} v_{P_{1}}^{\text {path }} v_{P_{2}}^{\text {path }}=v_{P_{1}}^{\text {cycle }} v_{P_{2}}^{\text {cycle }} .
\end{aligned}
$$

In particular, the second equality of (4.2) implies that

$$
v_{P_{1} P_{2}^{*}}^{\text {cycle }}=v_{a_{n+1}, a_{2}}^{a_{1}} v_{a_{n-1}, a_{m-1}}^{a_{n}} v_{P_{1}}^{\text {path }} v_{P_{2}^{*}}^{\text {path }} .
$$

Example 4.1 (product of $v$ along a path and a cycle product). Consider the graph $\Gamma=(V, E)$ where $V=\{a, b, c, d\}$ and $E=\{\{a, b\},\{a, d\},\{d, c\},\{b, c\},\{b, d\}\}$ and let $\mathbb{k}=\mathbb{C}$. Its associated double graph is the following quiver. 


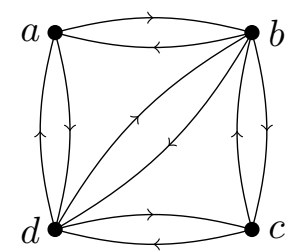

It is easy to show that the following are skew-zigzag coefficients for $\Gamma$ using straightforward calculations:

$$
\begin{aligned}
& v_{b, d}^{a}=v_{b, d}^{c}=2, \quad v_{d, b}^{a}=v_{d, b}^{c}=1 / 2, \quad v_{a, c}^{d}=v_{a, c}^{b}=5, \quad v_{c, a}^{d}=v_{c, a}^{b}=1 / 5, \quad v_{b, c}^{d}=v_{d, c}^{b}=7, \\
& v_{c, b}^{d}=v_{c, d}^{b}=1 / 7, \quad v_{a, b}^{d}=v_{a, d}^{b}=5 / 7, \quad v_{b, a}^{d}=v_{d, a}^{b}=7 / 5, \quad v_{y, y}^{x}=1 \quad \text { for all }\{x, y\} \in E .
\end{aligned}
$$

Now, consider the cycles $P_{1}=(d|b| c \mid d)$ and $P_{2}=(d|a| b \mid d)$. Then, we have

$$
v_{P_{1}}^{\text {path }}=14, \quad v_{P_{2}}^{\text {path }}=5 / 14, \quad v_{P_{1} P_{2}}^{\text {path }}=1, \quad v_{P_{1}}^{\text {cycle }}=2, \quad v_{P_{2}}^{\text {cycle }}=1 / 2, \quad \text { and } \quad v_{P_{1} P_{2}}^{\text {cycle }}=1 .
$$

Remark 4.2. We note that $A_{v}(\Gamma)$ is both a $\mathbb{k}$-algebra and a $\mathbb{k} \mathcal{Q}_{0}$-algebra. Moreover, note that a homomorphism of $\mathbb{k}_{\mathfrak{Q}} \mathcal{Q}_{0}$-modules is precisely a homomorphism of $\mathbb{k}$-modules that fixes the vertices.

Lemma 4.3. Let $\Gamma$ be a connected graph with at least 3 vertices and $v$ and $u$ be two collections of skew-zigzag coefficients. Suppose that

$$
\phi: A_{v}(\Gamma) \rightarrow A_{u}(\Gamma)
$$

is an isomorphism of graded algebras such that $\phi([a])=[a]$ for all $a \in V$. Then

$$
u_{P}^{\text {cycle }}=v_{P}^{\text {cycle }} \text {, }
$$

for any cycle $P$.

Proof. For all $\{a, b\} \in E$, we have $\phi([a \mid b])=\alpha_{a, b}[a \mid b]$ for some $\alpha_{a, b} \in \mathbb{k}^{*}$. In addition, for any $\{a, b\} \in E$ we have

$$
\phi([a|b| a])=\phi([a \mid b][b \mid a])=\phi([a \mid b]) \phi([b \mid a])=\alpha_{a, b}[a \mid b] \alpha_{b, a}[b \mid a]=\alpha_{a, b} \alpha_{b, a}[a|b| a] .
$$

Let $a, b, c \in V$ be such that $a$ is connected to both $b$ and $c$. Then

$$
\begin{aligned}
\alpha_{a, b} \alpha_{b, a} u_{b, c}^{a}[a|c| a] & =\alpha_{a, b} \alpha_{b, a}[a|b| a]=\alpha_{a, b} \alpha_{b, a}[a \mid b][b \mid a] \\
& =\phi([a \mid b][b \mid a])=\phi([a|b| a])=\phi\left(v_{b, c}^{a}[a|c| a]\right)=v_{b, c}^{a} \alpha_{a, c} \alpha_{c, a}[a|c| a]
\end{aligned}
$$

Thus, we have

$$
u_{b, c}^{a}=\frac{\alpha_{a, c} \alpha_{c, a}}{\alpha_{a, b} \alpha_{b, a}} v_{b, c}^{a} .
$$

Therefore, if $P=\left(a_{1}|\ldots| a_{n}\right)$ is a cycle, we have

$$
u_{P}^{\text {cycle }}=\frac{\alpha_{a_{1}, a_{n-1}} \alpha_{a_{n-1}, a_{1}}}{\alpha_{a_{1}, a_{2}} \alpha_{a_{2}, a_{1}}}\left(\prod_{i=2}^{n-2} \frac{\alpha_{a_{i}, a_{i-1}} \alpha_{a_{i-1}, a_{i}}}{\alpha_{a_{i}, a_{i+1}} \alpha_{a_{i+1}, a_{i}}}\right) \frac{\alpha_{a_{n-1}, a_{n-2}} \alpha_{a_{n-2}, a_{n-1}}}{\alpha_{a_{n-1}, a_{1}} \alpha_{a_{1}, a_{n-1}}} v_{P}^{\text {cycle }}=v_{P}^{\text {cycle }},
$$

as required.

The following proposition shows that the converse to Lemma 4.3 holds. 
Proposition 4.4. Let $\Gamma=(V, E)$ be a connected graph with at least 3 vertices and let $v$ and $u$ be two collections of skew-zigzag coefficients. Then $v_{P}^{\text {cycle }}=u_{P}^{\text {cycle }}$ for every cycle $P$ if and only if there exists an isomorphism of graded algebras $\phi: A_{v}(\Gamma) \stackrel{\cong}{\rightrightarrows} A_{u}(\Gamma)$ such that $\phi([a])=[a]$ for all $a \in V$.

Proof. $\Rightarrow$ : Fix an edge $\{a, b\} \in E$. For any $\{d, e\} \in E$, consider a path $P=\left(a_{1}|\ldots| a_{n}\right)$ with $a_{1}=a, a_{n-1}=d$ and $a_{n}=e$. Note that since $\Gamma$ is connected, there is at least one such path. Now, choose $\alpha_{d, e}=\alpha_{e, d} \in \mathbb{k}^{*}$ such that

$$
\alpha_{d, e}^{2}=u_{b, a_{2}}^{a} v_{a_{2}, b}^{a} u_{P}^{\text {path }} v_{P^{*}}^{\text {path }} .
$$

We will show that since we have $v_{C}^{\text {cycle }}=u_{C}^{\text {cycle }}$ for any cycle $C,(4.4)$ is independent of the choice of the path. Let $P_{1}=\left(a_{1}|\ldots| a_{n}\right)$ and $P_{2}=\left(b_{1}|\ldots| b_{m}\right)$ be two paths such that $a=a_{1}=b_{1}$, $d=a_{n-1}=b_{m-1}$ and $e=a_{n}=b_{m}$. Since $P_{1} P_{2}^{*}$ is a cycle, we have

$$
v_{P_{1} P_{2}^{*}}^{\text {cycle }}=v_{b_{2}, a_{2}}^{a_{1}} v_{a_{n-1}, b_{m-1}}^{a_{n}} v_{P_{1}}^{\text {path }} v_{P_{2}^{*}}^{\text {path }}=v_{b_{2}, b}^{a} v_{b, a_{2}}^{a} v_{P_{1}}^{\text {path }} v_{P_{2}^{*}}^{\text {path }},
$$

where the first equality follows from (4.3) and the second equality follows from Lemma 3.4 and the fact that $a_{n-1}=b_{m-1}$. Similarly, we obtain

$$
u_{P_{1} P_{2}^{*}}^{\text {cycle }}=u_{b_{2}, b}^{a} u_{b, a_{2}}^{a} u_{P_{1}}^{\text {path }} u_{P_{2}^{*}}^{\text {path }} .
$$

Since $v_{P_{1} P_{2}^{*}}^{\text {cycle }}=u_{P_{1} P_{2}^{*}}^{\text {cycle }}$, we have

$$
v_{b_{2}, b}^{a} v_{b, a_{2}}^{a} v_{P_{1}}^{\mathrm{path}} v_{P_{2}^{*}}^{\mathrm{path}}=u_{b_{2}, b}^{a} u_{b, a_{2}}^{a} u_{P_{1}}^{\mathrm{path}} u_{P_{2}^{*}}^{\mathrm{path}} .
$$

Consequently,

$$
u_{b, b_{2}}^{a} v_{b_{2}, b}^{a} u_{P_{2}}^{\text {path }} v_{P_{2}^{*}}^{\text {path }}=u_{b, a_{2}}^{a} v_{a_{2}, b}^{a} u_{P_{1}}^{\text {path }} v_{P_{1}^{*}}^{\text {path }},
$$

as required. Thus the coefficients $\alpha_{d, e}$ are path independent. Now, define a map $\phi$ as follows

$$
\phi: \quad \mathbb{k} D \Gamma \rightarrow \mathbb{k} D \Gamma, \quad(d) \mapsto(d) \quad \text { for all } d \in V, \quad(d \mid e) \mapsto \alpha_{d, e}(d \mid e) \quad \text { for all }\{d, e\} \in E .
$$

By definition this map is $\mathbb{k}$-linear. We then extend the map to longer paths by requiring it to be an algebra homomorphism. We will now show that $\phi\left(I_{v}\right) \subseteq I_{u}$. Now suppose $\{x, y\},\{x, z\} \in E$. Let $P_{1}=\left(a_{1}|\ldots| a_{n}\right)$ and $P_{2}=\left(b_{1}|\ldots| b_{m}\right)$ be two paths such that $a=a_{1}=b_{1}, x=a_{n-1}=$ $b_{m-1}, y=a_{n}$ and $z=b_{m}$. We have

$$
\frac{\alpha_{x, y}^{2}}{\alpha_{x, z}^{2}} v_{z, y}^{x}=\frac{u_{b, a_{2}}^{a} v_{a_{2}, b}^{a} u_{P_{1}}^{\text {path }} v_{P_{1}^{*}}^{\text {path }}}{u_{b, b_{2}}^{a} v_{b_{2}, b}^{a} u_{P_{2}}^{\text {path }} v_{P_{2}^{*}}^{\text {path }}} v_{z, y}^{x}=\frac{v_{a_{2}, b_{2}}^{a} v_{P_{1}^{*}}^{\text {path }} v_{P_{2}}^{\text {path }}}{u_{a_{2}, b_{2}}^{a} u_{P_{1}^{*}}^{\text {path }} u_{P_{2}}^{\text {path }}} v_{z, y}^{x} .
$$

The path $P_{2}(z|x| y) P_{1}^{*}$ is a cycle. Thus,

$$
v_{P_{2}(z|x| y) P_{1}^{*}}^{\text {cycle }}=v_{a_{2}, b_{2}}^{a} v_{P_{2}(z|x| y) P_{1}^{*}}^{\text {path }}=v_{a_{2}, b_{2}}^{a} v_{x, x}^{z} v_{x, x}^{y} v_{P_{2}}^{\text {path }} v_{(z|x| y)}^{\text {path }} v_{P_{1}^{*}}^{\text {path }}=v_{a_{2}, b_{2}}^{a} v_{P_{2}}^{\text {path }} v_{P_{1}^{*}}^{\text {path }} v_{z, y}^{x} \text {, }
$$

where the second equality uses (4.1). Similarly, we obtain

$$
u_{P_{2}(z|x| y) P_{1}^{*}}^{\text {cycle }}=u_{a_{2}, b_{2}}^{a} u_{P_{2}}^{\text {path }} u_{P_{1}^{*}}^{\text {path }} u_{z, y}^{x} \text {. }
$$

Since we have $v_{P_{2}(z|x| y) P_{1}^{*}}^{\text {cycle }}=u_{P_{2}(z|x| y) P_{1}^{*}}^{\text {cycle }}$, we must also have

$$
v_{a_{2}, b_{2}}^{a} v_{P_{2}}^{\text {path }} v_{P_{1}^{*}}^{\text {path }} v_{z, y}^{x}=u_{a_{2}, b_{2}}^{a} u_{P_{2}}^{\text {path }} u_{P_{1}^{*}}^{\text {path }} u_{z, y}^{x} \text {. }
$$


Combining (4.5) and (4.6) gives us

$$
\frac{\alpha_{x, y}^{2}}{\alpha_{x, z}^{2}} v_{z, y}^{x}=\frac{v_{a_{2}, b_{2}}^{a} v_{P_{1}^{*}}^{\text {path }} v_{P_{2}}^{\text {path }}}{u_{a_{2}, b_{2}}^{a} u_{P_{1}^{*}}^{\text {path }} u_{P_{2}}^{\text {path }}} v_{z, y}^{x}=\frac{u_{a_{2}, b_{2}}^{a} u_{z, y}^{x} u_{P_{1}^{*}}^{\text {path }} u_{P_{2}}^{\text {path }}}{u_{a_{2}, b_{2}}^{a} u_{P_{1}^{*}}^{\text {path }} u_{P_{2}}^{\text {path }}}=u_{z, y}^{x} .
$$

Hence, we can now deduce that $\phi\left(I_{v}\right) \subseteq I_{u}$, and thus $\phi$ induces an algebra homomorphism

$$
\bar{\phi}: A_{v}(\Gamma) \rightarrow A_{u}(\Gamma), \quad \begin{cases}{[d] \mapsto[d],} & d \in V, \\ {[d \mid e] \mapsto \alpha_{d, e}[d \mid e],} & \{d, e\} \in E \\ {[d|e| d] \mapsto \alpha_{d, e}^{2}[d|e| d],} & \{d, e\} \in E .\end{cases}
$$

Since $\phi$ is surjective, $\bar{\phi}$ is also surjective. Since $A_{v}(\Gamma)$ and $A_{u}(\Gamma)$ have the same dimension, $\bar{\phi}$ is also injective. Consequently, $\bar{\phi}$ is an isomorphism and hence $A_{v}(\Gamma) \cong A_{u}(\Gamma)$.

The converse of this proposition is Lemma 4.3.

We will now introduce the concept of graph cohomology. Let $\Gamma=(V, E)$ be a connected graph and $D \Gamma=\left(V, E^{\prime}\right)$ its double graph. Let

$$
\mathbb{Z} V=\left\{\sum_{a \in V} \alpha_{a} a \mid \alpha_{a} \in \mathbb{Z} \text { for all } a \in V\right\}, \quad \mathbb{Z} E^{\prime}=\left\{\sum_{e \in E^{\prime}} \alpha_{e} e \mid \alpha_{e} \in \mathbb{Z} \text { for all } e \in E^{\prime}\right\} .
$$

Define the map $\delta$ by

$$
\delta: \mathbb{Z} E^{\prime} /\{(a \mid b)+(b \mid a) \mid\{a, b\} \in E\} \rightarrow \mathbb{Z} V, \quad e \mapsto s(e)-t(e),
$$

where we extend the map by linearity. For any path $\left(a_{1}|\ldots| a_{n}\right)$, we associate the element $\sum_{i=1}^{n-1}\left(a_{i} \mid a_{i+1}\right) \in \mathbb{Z} E^{\prime}$ to it. Notice that if $a_{1}=a_{n}$, then $\delta\left(\sum_{i=1}^{n-1}\left(a_{i} \mid a_{i+1}\right)\right)=0$. Let $\mathcal{C}=\operatorname{ker} \delta$. We call $\mathcal{C}$ the space of cycles of $\Gamma$. The space of cycles is a $\mathbb{Z}$-submodule of the free $\mathbb{Z}$-module $\mathbb{Z} E^{\prime} /\{(a \mid b)+(b \mid a) \mid\{a, b\} \in E\}$ and thus it is a free $\mathbb{Z}$-module. Consequently, it has a $\mathbb{Z}$-basis.

Lemma 4.5 ([8, Section 4.4]). Let $\Gamma=(V, E)$ be a connected graph. Then $\operatorname{rank}(\mathcal{C})=|E|-$ $|V|+1$.

Let $T=\left(V_{T}, E_{T}\right)$ be a spanning tree of a graph $\Gamma=(V, E)$ and let $e \in V \backslash V_{T}$. We shall denote by $T+e$ the subgraph of $\Gamma$ with vertex set $V$ and edge set $E_{T} \cup\{e\}$. Moreover, for any cycle $C=\left(a_{1}|\ldots| a_{n}=a_{1}\right)$, we say that an edge $(a \mid b)$ is in $C$ if $\left.(a \mid b) \in\left\{\left(a_{i} \mid a_{i+1}\right)\right\} \mid 1 \leq i \leq n-1\right\}$.

Lemma 4.6. Let $\Gamma=(V, E)$ be a connected graph with $|V|=n$ and $|E|=m$. There exists a basis of $\mathcal{C}, \mathcal{B}=\left\{C_{1}, \ldots, C_{m-n+1}\right\}$, such that, for all $1 \leq i \leq m-n+1$, there exists $\left(b_{i} \mid c_{i}\right)$ in $C_{i}$ such that $\left(b_{i} \mid c_{i}\right)$ is not in $C_{k}$ for $k \neq i$.

Proof. Let $\Gamma=(V, E)$ be a connected graph with $|V|=n$ and $|E|=m$. Pick a spanning tree $T=\left(V_{T}, E_{T}\right)$ of $\Gamma$. Let $E \backslash E_{T}=\left\{e_{1}, \ldots, e_{m-n+1}\right\}$. Notice that for each $i \in\{1, \ldots, m-n+1\}$, $T+e_{i}$ contains one simple cycle. Thus, the quiver $D\left(T+e_{i}\right)$ contains two corresponding simple cycles, $C_{i}$ and $-C_{i}$. Let

$$
\mathcal{B}=\left\{C_{i} \mid i=1, \ldots, m-n+1\right\} .
$$

Clearly the elements of $\mathcal{B}$ are linearly independent. Moreover, $|\mathcal{B}|=m-n+1$. Thus, by Lemma $4.5, \mathcal{B}$ is a $\mathbb{Q}$-basis of $\mathcal{C} \otimes_{\mathbb{Z}} \mathbb{Q}$. But since the coefficient of $e_{i}$ in $C_{i}$ is one, it follows that $\mathcal{B}$ is a $\mathbb{Z}$-basis of $\mathcal{C}$. Finally, it is also clear that for all $i=1, \ldots, m-n+1$ we have $\left(b_{i} \mid c_{i}\right)$ in $C_{k}$ if and only if $k=i$, where $\left(b_{i} \mid c_{i}\right)$ is the corresponding directed edge of $e_{i}$ in $C_{i}$. 
Let $\Gamma=(V, E)$ be a connected graph and $D \Gamma=\left(V, E^{\prime}\right)$ be its double graph. The graph cohomology of $\Gamma$ is defined to be the space of group homomorphisms from $\mathcal{C}$ to $\mathbb{k}^{*}$ :

$$
H^{1}\left(\Gamma, \mathbb{k}^{*}\right)=\operatorname{Hom}_{\text {group }}\left(\mathcal{C}, \mathbb{k}^{*}\right) .
$$

Note that the operation in this group is pointwise multiplication.

Let $z \in \mathcal{C}$. Take a representative $\sum_{e \in E^{\prime}} \alpha_{e} e$ of $z$ in $\mathbb{N} E^{\prime}=\left\{\sum_{e \in E^{\prime}} \alpha_{e} e \mid \alpha_{e} \in \mathbb{N}\right.$ for all $\left.e \in E^{\prime}\right\}$. Since $z \in \mathcal{C}=\operatorname{ker} \delta$, for each $a \in V$ we have $\sum_{e \in S} \alpha_{e}=\sum_{e \in T} \alpha_{e}$ where $S=\left\{e \in E^{\prime} \mid s(e)=a\right\}$ and $T=\left\{e \in E^{\prime} \mid t(e)=a\right\}$. Thus, we can choose $n_{a} \in \mathbb{N}$ and vertices $b_{a, 1}, \ldots, b_{a, n_{a}}, c_{a, 1}, \ldots, c_{a, n_{a}}$ such that

$$
\sum_{a \in V} \sum_{i=1}^{n_{a}}\left(\left(b_{a, i} \mid a\right)+\left(a \mid c_{a, i}\right)\right)=2 z .
$$

Now, for a collection of skew-zigzag coefficients $v$, define

$$
f_{v, a}(z)=\prod_{i=1}^{n_{a}} v_{b_{a, i}, c_{a, i}}^{a} .
$$

Lemma 4.7. Let $\Gamma=(V, E)$ be a connected graph, $v$ be a collection of skew-zigzag coefficients, $a \in V$ and $z \in \mathcal{C}$. Set $b_{a, 1}, \ldots, b_{a, n_{a}}$ and $c_{a, 1}, \ldots, c_{a, n_{a}}$ as in the previous paragraph. Then, $f_{v, a}(z)$ is independent of the order chosen for $b_{a, 1}, \ldots, b_{a, n_{a}}$ and $c_{a, 1}, \ldots, c_{a, n_{a}}$ and $f_{v, a}(z)$ is independent of the representative of $z$ chosen in $\mathbb{N} E^{\prime}$.

Proof. For the first claim, it suffices to show that $f_{v, a}(z)$ remains unchanged when we interchange $c_{a, j}$ and $c_{a, j+1}$ for some $j \in\left\{1, \ldots, n_{a}-1\right\}$. Indeed, we have

$$
\begin{aligned}
& v_{b_{a, j}, c_{a, j+1}}^{a} v_{b_{a, j+1}, c_{a, j}}^{a} \prod_{\substack{i=1 \\
i \neq j, j+1}}^{n_{a}} v_{b_{a, i}, c_{a, i}}^{a} \\
& \quad=v_{b_{a, j}, c_{a, j}}^{a} v_{c_{a, j}, c_{a, j+1}}^{a} v_{b_{a, j+1}, c_{a, j+1}}^{a} v_{c_{a, j+1}, c_{a, j}}^{a} \prod_{\substack{i=1 \\
i \neq j, j+1}}^{n_{a}} v_{b_{a, i}, c_{a, i}}^{a}=\prod_{i=1}^{n_{a}} v_{b_{a, i}, c_{a, i}}^{a},
\end{aligned}
$$

where the second equality follows from Lemma 3.4 and the third equality follows from the second condition of Definition 3.1.

For the second part of the lemma, it suffices to show that $f_{v, a}(z)$ remains unchanged when you remove $b_{a, n}$ and $c_{a, n}$ when $b_{a, n}=c_{a, n}$. But this is obvious as $v_{b_{a, n}, c_{a, n}}^{a}=v_{b_{a, n}, b_{a, n}}^{a}=1$.

Now, for any collection of skew-zigzag coefficients, $v$, define the map $f_{v}$ to be

$$
f_{v}: \mathcal{C} \rightarrow \mathbb{k}^{*}, \quad z \mapsto \prod_{a \in V} f_{v, a}(z)
$$

It is clear by the definition of $f_{v}$ that we have $f_{v}\left(z_{1}+z_{2}\right)=f_{v}\left(z_{1}\right) f_{v}\left(z_{2}\right)$ for any $z_{1}, z_{2} \in \mathcal{C}$. Thus, $f_{v} \in H^{1}\left(\Gamma, \mathbb{k}^{*}\right)$. In addition, if $C$ is a cycle in $D \Gamma$, then it is clear that $f_{v}(C)=v_{C}^{\text {cycle }}$.

Let $\mathrm{SZC}=\{v \mid v$ is a collection of skew-zigzag coefficients $\}$. For $u, v \in \mathrm{SZC}$, define $u \cdot v$ to be the set of skew-zigzag coefficients defined as follows:

$$
(u \cdot v)_{b, c}^{a}=u_{b, c}^{a} v_{b, c}^{a}
$$


for all $\{a, b\},\{a, c\} \in E$. It is straightforward to check that this introduces a group structure on SZC. We then define an equivalence relation on SZC as follows:

$$
\begin{aligned}
v \equiv u \Longleftrightarrow & \text { there exists an isomorphism } \phi: A_{v}(\Gamma) \rightarrow A_{u}(\Gamma) \\
& \text { such that } \phi([a])=[a] \text { for all } a \in V .
\end{aligned}
$$

By Lemma 4.3 and Proposition 4.4, we have

$$
v \equiv u \Longleftrightarrow v_{P}^{\text {cycle }}=u_{P}^{\text {cycle }} \text { for every cycle } P \text { in } \Gamma \text {. }
$$

Now let

$$
\Sigma=\left\{A_{v}(\Gamma) \mid v \in \mathrm{SZC}\right\} .
$$

Let $\sim$ be the equivalence relation on $\Sigma$ defined by

$$
\begin{aligned}
A_{v}(\Gamma) \sim A_{u}(\Gamma) \Longleftrightarrow & \text { there exists an isomorphism } \phi: A_{v}(\Gamma) \rightarrow A_{u}(\Gamma) \\
& \text { such that } \phi([a])=[a] \forall a \in V .
\end{aligned}
$$

Let $v \in \mathrm{SZC}$. From now on, we shall use $[v]$ to denote the equivalence class of $v$ in $\mathrm{SZC} / \equiv$. Furthermore, we shall use $\left[A_{v}(\Gamma)\right]_{\sim}$ and $\left[A_{v}(\Gamma)\right] \cong$ to denote the equivalence classes of $A_{v}(\Gamma)$ in $\Sigma / \sim$ and $\Sigma / \cong$ respectively, where $\cong$ denotes isomorphism of graded algebras. Note that $\Sigma / \sim$ and $\mathrm{SZC} / \equiv$ are naturally isomorphic sets via the map

$$
\phi: \Sigma / \sim \rightarrow \mathrm{SZC} / \equiv, \quad\left[A_{v}(\Gamma)\right]_{\sim} \mapsto[v] .
$$

Theorem 4.8. Let $\Gamma=(V, E)$ be a connected graph. Then we have

$$
\Sigma / \sim \cong \mathrm{SZC} / \equiv \cong H^{1}\left(\Gamma, \mathbb{k}^{*}\right),
$$

where the first isomorphism is an isomorphism of sets and the second isomorphism is an isomorphism of groups.

Proof. Define the map $\psi$ by

$$
\psi: \mathrm{SZC} \rightarrow H^{1}\left(\Gamma, \mathbb{k}^{*}\right), \quad v \mapsto f_{v} .
$$

It is clear that this map is a group homomorphism. Let $v, u \in \mathrm{SZC}$. Then, we have

$$
\begin{aligned}
\psi(v)=\psi(u) & \Longleftrightarrow f_{v}=f_{u} \Longleftrightarrow f_{v}(P)=f_{u}(P) \text { for all } P \in \mathcal{C} \\
& \Longleftrightarrow v_{P}^{\text {cycle }}=u_{P}^{\text {cycle }} \text { for all } P \in \mathcal{C} \Longleftrightarrow v \equiv u .
\end{aligned}
$$

Hence, $\mathrm{SZC} / \operatorname{ker} \psi=\mathrm{SZC} / \equiv$.

Let us now prove that $\psi$ is surjective. By Lemma 4.6, there exists a basis of cycles $\mathcal{B}=$ $\left\{C_{1}, \ldots, C_{|E|-|V|+1}\right\}$ of $\mathcal{C}$ such that, for all $1 \leq i \leq|E|-|V|+1$, there exists $\left(b_{i} \mid c_{i}\right) \in C_{i}$ such that $\left(b_{i} \mid c_{i}\right) \in C_{k}$ if and only if $k=i$.

Let $f$ be a group homomorphism from $\mathcal{C}$ to $\mathbb{k}^{*}$. We define coefficients as follows:

$$
\begin{aligned}
& v_{b, b}^{a}=1 \text { for all }\{a, b\} \in E, \\
& v_{b, c}^{a}=1 \text { for all }\{a, b\},\{a, c\} \in E, \quad a \neq b_{i} \text { for all } i=1, \ldots,|E|-|V|+1 .
\end{aligned}
$$

Then, for all $i=1, \ldots,|E|-|V|+1$ we define

$$
\begin{aligned}
& 1 / v_{c_{i}, a}^{b_{i}}=v_{a, c_{i}}^{b_{i}}=f\left(C_{i}\right) \text { for all } a \neq c_{k}, 1 \leq k \leq|E|-|V|+1, \\
& v_{c_{k}, c_{i}}^{b_{i}}=f\left(C_{i}\right) / f\left(C_{k}\right) \text { for all } k \in\{1, \ldots,|E|-|V|+1\} \text { such that } b_{i}=b_{k}, \\
& v_{a, c}^{b_{i}}=1 \text { for all }\left\{b_{i}, a\right\},\left\{b_{i}, c\right\} \in E, a \neq c_{i} \neq c .
\end{aligned}
$$


It is clear that the set $v=\left(v_{b, c}^{a} \mid\{a, b\},\{a, c\} \in E\right)$ is a collection of skew-zigzag coefficients. Moreover, since $\left(b_{i} \mid c_{i}\right)$ is in $C_{k}$ if and only if $k=i$ for all $i=1, \ldots,|E|-|V|+1$, it is also clear that $f_{v}\left(C_{i}\right)=f\left(C_{i}\right)$ for all $i=1, \ldots,|E|-|V|+1$. Thus, we must have $\psi(v)=f_{v}=f$. Consequently, $\psi$ is surjective.

Since $\psi$ is surjective, the first isomorphism theorem implies $\mathrm{SZC} / \equiv \cong H^{1}\left(\Gamma, \mathbb{k}^{*}\right)$. The result then follows using (4.7).

Remark 4.9. In [5, Section 4], the authors state that "the moduli space of skew-zigzag algebras is naturally isomorphic to $H^{1}\left(\Gamma, \mathbb{C}^{*}\right)$ ". In light of Theorem 4.8 , we assume that the moduli space they had in mind was $\Sigma / \sim$.

Let $\Gamma=(V, E)$ be a graph. We say that a permutation $\sigma$ of the elements of $V$ is a graph automorphism of $\Gamma$ if, for all $a, b \in V$, we have

$$
\{a, b\} \in E \Longleftrightarrow\{\sigma(a), \sigma(b)\} \in E .
$$

The identity permutation is called the trivial graph automorphism. A graph is said to be asymmetric if it admits only the trivial graph automorphism, otherwise it is said to be symmetric.

Suppose $\sigma$ is a graph automorphism of $\Gamma$. For any path $P=\left(a_{1}|\ldots| a_{n}\right)$ we let $\sigma(P)=$ $\left(\sigma\left(a_{1}\right)|\ldots| \sigma\left(a_{n}\right)\right)$. Notice that if $P$ is a cycle, then $\sigma(P)$ is also a cycle. Moreover, if $P_{1}$ and $P_{2}$ are two paths, then it is clear that $\sigma\left(P_{1} P_{2}\right)=\sigma\left(P_{1}\right) \sigma\left(P_{2}\right)$. Thus, the map $\sigma$ induces an automorphism of the path algebra $\mathbb{k} D \Gamma$

$$
\phi_{\sigma}: \mathbb{k} D \Gamma \rightarrow \mathbb{k} D \Gamma, \quad P \mapsto \sigma(P) .
$$

Fix a collection of skew-zigzag coefficients $v$. We define $(\sigma v)_{b, c}^{a}$ for all $\{a, b\},\{a, c\} \in E$ as follows:

$$
(\sigma v)_{b, c}^{a}=v_{\sigma^{-1}(b), \sigma^{-1}(c)}^{\sigma^{-1}(a)} .
$$

Then we define $\sigma v=\left((\sigma v)_{b, c}^{a} \mid\{a, b\},\{a, c\} \in E\right)$. The map $(\sigma, v) \mapsto \sigma v$ defines an action of the group of graph automorphisms on the set of skew-zigzag coefficients.

It is straightforward to verify that $\sigma\left(I_{v}\right)=I_{\sigma v}$. Thus we obtain an isomorphism

$$
\sigma: A_{v}(\Gamma) \rightarrow A_{\sigma v}(\Gamma), \quad[P] \mapsto[\sigma(P)] .
$$

Let

$$
\psi: A_{v}(\Gamma) \rightarrow A_{u}(\Gamma)
$$

be an isomorphism of graded algebras where $v$ and $u$ are two collections of skew-zigzag coefficients. Suppose that $V=\left\{a_{1}, \ldots, a_{n}\right\}$. Although it follows from more advance concepts, we will use an elementary approach to prove that vertices must be mapped to vertices. For all $1 \leq i \leq n$, let $\psi\left(\left[a_{i}\right]\right)=\sum_{j=1}^{n} \alpha_{i j}\left[a_{j}\right]$, where $\alpha_{i j} \in \mathbb{k}$ for all $1 \leq i, j \leq n$. For any two vertices $a_{i}, a_{k} \in V, 1 \leq i, k \leq n, i \neq k$, we have

$$
0=\psi(0)=\psi\left(\left[a_{i}\right]\left[a_{k}\right]\right)=\left(\sum_{j=1}^{n} \alpha_{i j}\left[a_{j}\right]\right)\left(\sum_{j=1}^{n} \alpha_{k j}\left[a_{j}\right]\right)=\sum_{j=1}^{n} \alpha_{i j} \alpha_{k j}\left[a_{j}\right] .
$$

Thus, for any $j=1, \ldots, n$, at least one of $\alpha_{i j}$ or $\alpha_{k j}$ is 0 . Hence, if $\alpha_{i j} \neq 0$ for some $1 \leq i, j \leq n$, then $\alpha_{k j}=0$ for all $1 \leq k \leq n, k \neq i$. Moreover, since $\psi$ is injective, $\psi\left(\left[a_{i}\right]\right) \neq 0$ for all $i=1, \ldots, n$, and so there exists $j_{i} \in\{1, \ldots, n\}$ such that $\alpha_{i j_{i}} \neq 0$ and thus $\alpha_{k j_{i}}=0$ for all 
$1 \leq k \leq n, k \neq i$. Therefore, for any $1 \leq i, k \leq n, i \neq k$ we have $j_{i} \neq j_{k}$. As a result, we must have $\alpha_{i j}=0$ for all $1 \leq i, j \leq n, j \neq j_{i}$. Consequently, we must have $\psi\left(\left[a_{i}\right]\right)=\alpha_{i j_{i}}\left[a_{j_{i}}\right]$ for some $j_{i} \in\{1, \ldots, n\}$. Furthermore, notice that we have

$$
\phi\left(\left[a_{i}\right]\right)=\phi\left(\left[a_{i}\right]\left[a_{i}\right]\right)=\phi\left(\left[a_{i}\right]\right) \phi\left(\left[a_{i}\right]\right)=\alpha_{i j_{i}} \alpha_{i j_{i}}\left[a_{j_{i}}\right]=\alpha_{i j_{i}} \phi\left(\left[a_{i}\right]\right),
$$

for all $1 \leq i \leq n$. Hence we must have $\alpha_{i j_{i}}=1$. Therefore, any isomorphism $\psi: A_{v}(\Gamma) \rightarrow A_{u}(\Gamma)$ induces a graph automorphism

$$
\sigma_{\psi}: V \rightarrow V, \quad a_{i} \mapsto a_{j_{i}}
$$

Then, we obtain the isomorphism

$$
\sigma_{\psi}^{-1} \circ \psi: A_{v}(\Gamma) \rightarrow A_{\sigma^{-1} u}(\Gamma)
$$

Therefore

$$
\psi=\sigma_{\psi} \circ\left(\sigma_{\psi}^{-1} \circ \psi\right), \quad \text { where } \sigma_{\psi}^{-1} \circ \psi([a])=[a] \text { for all } a \in V .
$$

Lemma 4.10. Let $\Gamma$ be a connected asymmetric graph with at least three vertices, and let $v$ and $u$ be two collections of skew-zigzag coefficients. Then $A_{v}(\Gamma) \cong A_{u}(\Gamma)$ as graded algebras if and only if $v \equiv u$.

Proof. Let $\Gamma$ be a connected asymmetric graph with at least three vertices, and $v$ and $u$ be two collections of skew-zigzag coefficients. Suppose that we have an isomorphism $\phi: A_{v}(\Gamma) \rightarrow A_{u}(\Gamma)$. Then in particular the map

$$
\sigma_{\phi}: V \rightarrow V, \quad a \mapsto s(\phi([a]))
$$

is a graph automorphism. Since $\Gamma$ is asymmetric, we must have $\sigma_{\phi}(a)=a$ for all $a \in V$. Thus $\phi([a])=[a]$ for all $a \in V$. Hence $v \equiv u$ by Lemma 4.3.

The reverse implication follows from the definition of the equivalence relation.

Let $\operatorname{Aut}(\Gamma)$ be the group of graph automorphisms of $\Gamma$. We define a group action of Aut $(\Gamma)$ on $H^{1}\left(\Gamma, \mathbb{k}^{*}\right)$ by

$$
(\sigma f)(C)=f\left(\sigma^{-1}(C)\right), \quad \sigma \in \operatorname{Aut}(\Gamma), \quad f \in H^{1}\left(\Gamma, \mathbb{k}^{*}\right), \quad C \in \mathcal{C} .
$$

For any $\sigma \in \operatorname{Aut}(\Gamma)$ and any collection of skew-zigzag coefficients $v$ we have an isomorphism given by (4.8) $\sigma: A_{v}(\Gamma) \rightarrow A_{\sigma v}(\Gamma)$. It is clear that $A_{v}(\Gamma) \mapsto A_{\sigma v}(\Gamma)$ defines a group action of $\operatorname{Aut}(\Gamma)$ on $\Sigma$ and that it preserves the equivalence relation $\sim$. Therefore, we have an induced action of $\operatorname{Aut}(\Gamma)$ on $\Sigma / \sim$, given by $X \mapsto \sigma X$ for all $X \in \Sigma / \sim$, where $\sigma X=\{\sigma x \mid x \in X\}$.

Lemma 4.11. For any graph $\Gamma$ we have the following isomorphism of sets

$$
(\Sigma / \sim) / \operatorname{Aut}(\Gamma) \cong \Sigma / \cong .
$$

Proof. Define

$$
\alpha: \Sigma / \sim \rightarrow \Sigma / \cong, \quad\left[A_{v}(\Gamma)\right]_{\sim} \mapsto\left[A_{v}(\Gamma)\right]_{\cong} .
$$

Clearly this is a well-defined surjective map. Moreover, notice that if $v$ and $u$ are two collections of skew-zigzag coefficients, then we have

$$
\begin{aligned}
\alpha\left(\left[A_{v}(\Gamma)\right]_{\sim}\right)=\alpha\left(\left[A_{u}(\Gamma)\right]_{\sim}\right) & \Longleftrightarrow\left[A_{v}(\Gamma)\right]_{\cong}=\left[A_{u}(\Gamma)\right]_{\cong} \\
& \Longleftrightarrow \text { there exists an isomorphism } \Phi: A_{v}(\Gamma) \rightarrow A_{u}(\Gamma) .
\end{aligned}
$$


Recall from (4.9) that every isomorphism between skew-zigzag algebras can be written as the composition of a graph automorphism and an isomorphism that fixes the vertices. Thus, there exists $\sigma \in \operatorname{Aut}(\Gamma)$ and an isomorphism $\gamma$ that fixes the vertices such that

$$
\Phi=\sigma \circ \gamma: A_{v}(\Gamma) \stackrel{\gamma}{\rightarrow} A_{\sigma^{-1}}(\Gamma) \stackrel{\sigma}{\rightarrow} A_{u}(\Gamma) .
$$

Since $\gamma$ fixes the vertices, we have $\left[A_{v}(\Gamma)\right]_{\sim}=\left[A_{\sigma^{-1} u}(\Gamma)\right]_{\sim}$ and so,

$$
\sigma\left[A_{v}(\Gamma)\right]_{\sim}=\sigma\left[A_{\sigma^{-1} u}(\Gamma)\right]_{\sim}=\left[A_{u}(\Gamma)\right]_{\sim} .
$$

Now, let $v$ and $u$ be two collections of skew-zigzag coefficients such that there exists $\sigma \in \operatorname{Aut}(\Gamma)$ such that $\sigma\left[A_{v}(\Gamma)\right]_{\sim}=\left[A_{u}(\Gamma)\right]_{\sim}$. Then, since $A_{\sigma^{-1} u}(\Gamma) \in\left[A_{v}(\Gamma)\right]_{\sim}$, there exists an isomorphism $\gamma$ that fixes the vertices such that we have the following

$$
A_{v}(\Gamma) \stackrel{\gamma}{\rightarrow} A_{\sigma^{-1} u}(\Gamma) \stackrel{\sigma}{\rightarrow} A_{u}(\Gamma) .
$$

Since $\sigma \circ \gamma$ is an isomorphism, we must have $\left[A_{v}(\Gamma)\right] \cong=\left[A_{u}(\Gamma)\right] \cong$ and thus, $\alpha\left(\left[A_{v}(\Gamma)\right]_{\sim}\right)=$ $\alpha\left(\left[A_{u}(\Gamma)\right]_{\sim}\right)$.

Consequently, for any $X_{1}, X_{2} \in \Sigma / \sim$, we have

$$
\alpha\left(X_{1}\right)=\alpha\left(X_{2}\right) \Longleftrightarrow \text { there exists } \sigma \in \operatorname{Aut}(\Gamma) \text { such that } \sigma X_{1}=X_{2} .
$$

Hence, we finally obtain $(\Sigma / \sim) / \operatorname{Aut}(\Gamma) \cong \Sigma / \cong$.

Theorem 4.12. For any graph $\Gamma$ we have the following isomorphism of sets

$$
\Sigma / \cong \cong H^{1}\left(\Gamma, \mathbb{k}^{*}\right) / \operatorname{Aut}(\Gamma) \text {. }
$$

Proof. By Theorem 4.8, we know that the map

$$
\psi \circ \phi: \Sigma / \sim \rightarrow H^{1}\left(\Gamma, \mathbb{k}^{*}\right), \quad\left[A_{v}(\Gamma)\right]_{\sim} \mapsto f_{v},
$$

is an isomorphism, where the map $\phi$ is as in (4.7) and $\psi: \mathrm{SZC} / \equiv \rightarrow H^{1}\left(\Gamma, \mathbb{k}^{*}\right),[v] \mapsto f_{v}$. Let us now prove that it preserves the $\operatorname{Aut}(\Gamma)$-action, i.e., for all $\sigma \in \operatorname{Aut}(\Gamma)$ and $v \in \mathrm{SZC}$, we have $(\psi \circ \phi)\left(\sigma\left[A_{v}(\Gamma)\right]_{\sim}\right)=\sigma(\psi \circ \phi)\left(\left[A_{v}(\Gamma)\right]_{\sim}\right)$. We have

$$
(\psi \circ \phi)\left(\sigma\left[A_{v}(\Gamma)\right]_{\sim}\right)=\psi([\sigma v])=f_{\sigma v},
$$

and

$$
\sigma(\psi \circ \phi)\left(\left[A_{v}(\Gamma)\right]_{\sim}\right)=\sigma(\psi([v]))=\sigma\left(f_{v}\right) .
$$

Let $C=\left(a_{1}|\ldots| a_{n}\right) \in C$ be a cycle. Then,

$$
\begin{aligned}
\sigma\left(f_{v}\right)(C)=f_{v}\left(\sigma^{-1}(C)\right) & =f_{v}\left(\left(\sigma^{-1}\left(a_{1}\right)|\ldots| \sigma^{-1}\left(a_{n}\right)\right)\right) \\
& =v_{\sigma^{-1}\left(a_{n-1}\right), \sigma^{-1}\left(a_{2}\right)}^{\sigma^{-1}\left(a_{1}\right)} \cdots v_{\sigma^{-1}\left(a_{n-2}\right), \sigma^{-1}\left(a_{n}\right)}^{\sigma^{-1}\left(a_{n-1}\right)}=f_{\sigma v}(C) .
\end{aligned}
$$

Hence $\sigma\left(f_{v}\right)=f_{\sigma v}$. Consequently, we must have $(\psi \circ \phi)\left(\sigma\left[A_{v}(\Gamma)\right]_{\sim}\right)=\sigma(\psi \circ \phi)\left(\left[A_{v}(\Gamma)\right]_{\sim}\right)$. So $\psi \circ \phi$ is an $\operatorname{Aut}(\Gamma)$-set isomorphism and thus,

$$
(\Sigma / \sim) / \operatorname{Aut}(\Gamma) \cong H^{1}\left(\Gamma, \mathbb{k}^{*}\right) / \operatorname{Aut}(\Gamma) .
$$

Lemma 4.11 then yields $\Sigma / \cong \cong H^{1}\left(\Gamma, \mathbb{k}^{*}\right) / \operatorname{Aut}(\Gamma)$.

In $[5$, Section 4] the authors state the following result without proof. 
Corollary 4.13. If $\Gamma$ is a tree then all of its skew-zigzag algebras are isomorphic.

Proof. The follows immediately from Theorem 4.12 and the fact that trees have trivial graph cohomology.

Example 4.14. Consider the graph $\Gamma=(V, E)$ given by $V=\{a, b, c\}$ and $E=\{\{a, b\},\{b, c\}$, $\{a, c\}\}$. The associated double graph is

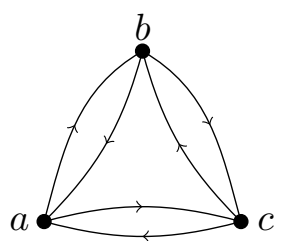

By Proposition 4.4, if $u, v \in \mathrm{SZC}$, then $A_{u}(\Gamma) \sim A_{v}(\Gamma)$ if and only if $u_{P}^{\text {cycle }}=v_{P}^{\text {cycle }}$ for every cycle $P$. Furthermore, recall that by (4.9), if $u, v \in \mathrm{SZC}$ are such that $A_{v}(\Gamma) \cong A_{u}(\Gamma)$ via an isomorphism $\phi$, then $\phi$ can be written as the composition of a graph automorphism $\sigma$ with an isomorphism $\gamma$ that fixes the vertices:

$$
\sigma \circ \gamma: A_{v}(\Gamma) \stackrel{\gamma}{\rightarrow} A_{\sigma^{-1}}(\Gamma) \stackrel{\sigma}{\rightarrow} A_{u}(\Gamma) .
$$

Thus, if $P$ is a cycle, then $v_{P}^{\text {cycle }}=u_{\sigma^{-1}(P)}^{\text {cycle }}$. Conversely, if there exists $\sigma \in \operatorname{Aut}(\Gamma)$, such that $v_{P}^{\text {cycle }}=u_{\sigma^{-1}(P)}^{\text {cycle }}$ for every cycle $P$, then we have $A_{v}(\Gamma) \cong A_{\sigma^{-1} u}(\Gamma)$ by Proposition 4.4 and $A_{\sigma^{-1} u}(\Gamma) \cong A_{u}(\Gamma)$ by (4.8). Thus, $A_{v}(\Gamma) \cong A_{u}(\Gamma)$. Consequently, $A_{v}(\Gamma) \cong A_{u}(\Gamma)$ if and only if there exists some $\sigma \in \operatorname{Aut}(\Gamma)$ such that $v_{P}^{\text {cycle }}=u_{\sigma^{-1}(P)}^{\text {cycle }}$ for all cycles $P$. Therefore, in order to compute $\Sigma / \sim$ and $\Sigma / \cong$, it suffices to consider the cycle products of $u$ and $v(u, v \in \mathrm{SZC})$ along every cycle.

It is clear that the $C=(a|b| c \mid a)$ yields a basis for the cycle space $\mathcal{C}$. So, if $u, v \in \mathrm{SZC}$, then $A_{u}(\Gamma) \sim A_{v}(\Gamma)$ if and only if $u_{C}^{\text {cycle }}=v_{C}^{\text {cycle }}$ and $A_{u}(\Gamma) \cong A_{v}(\Gamma) \Longleftrightarrow u_{C}^{\text {cycle }}=v_{C}^{\text {cycle }}$ or $u_{C^{*}}^{\text {cycle }}=v_{C}^{\text {cycle }}$.

Let $\mathbb{k}=\mathbb{C}$ and $x R y \Longleftrightarrow x=y$ or $x^{-1}=y\left(x, y \in \mathbb{C}^{*}\right)$. Consider the maps

$$
\phi: \Sigma / \sim \rightarrow \mathbb{C}^{*}, \quad\left[A_{v}(\Gamma)\right]_{\sim} \mapsto v_{C}^{\text {cycle }}, \quad \psi: \Sigma / \cong \rightarrow \mathbb{C}^{*} / R, \quad\left[A_{v}(\Gamma)\right]_{\cong} \mapsto v_{C}^{\text {cycle }} .
$$

These maps are well-defined and injective by the above remark. Surjectivity follows from the fact that if $x \in \mathbb{C}^{*}$, then the following is a collection of skew-zigzag coefficients:

$$
\begin{aligned}
& v_{b, b}^{a}=v_{c, c}^{a}=v_{a, a}^{b}=v_{c, c}^{b}=v_{a, a}^{c}=v_{b, b}^{c}=v_{b, c}^{a}=v_{c, b}^{a}=v_{a, c}^{b}=v_{c, a}^{b}=1, \\
& v_{a, b}^{c}=x, \quad v_{b, a}^{c}=x^{-1} .
\end{aligned}
$$

Thus, $\Sigma / \sim \cong \mathbb{C}^{*}$, whereas $\Sigma / \cong \cong \mathbb{C}^{*} / R$. Note that, $\mathbb{C}^{*} ¥ \mathbb{C}^{*} / R$ since, in $\mathbb{C}^{*} / R$, every element is its own inverse which is not true in $\mathbb{C}^{*}$.

\section{Other constructions of some skew-zigzag algebras}

We conclude with a discussion of other constructions of certain skew-zigzag algebras that have appeared in the literature $[2,3]$.

Let $\Gamma=(V, E)$ be a connected graph. We call $\Omega=\left(\epsilon_{a, b} \in \mathbb{k}^{*} \mid a, b \in V\right)$ a collection of orientation coefficients if, for any pair of vertices $a, b \in V$, we have $\epsilon_{a, b}=0$ if $\{a, b\} \notin E$ and $\epsilon_{a, b}=-\epsilon_{b, a}$ if $\{a, b\} \in E$. If $\Gamma$ has at least 3 vertices, we define the algebra $B_{\Omega}^{\Gamma}$ to be the quotient algebra of the path algebra of $D \Gamma$ by the two sided ideal $I_{\Omega}$ generated by elements of the form 
- $(a|b| c)$ for $\{a, b\},\{b, c\} \in E$ and $a \neq c$, and

- $\epsilon_{a, b}(a|b| a)-\epsilon_{a, c}(a|c| a)$ such that $a$ is connected to both $b$ and $c$.

Lemma 5.1. Let $\Gamma$ be a connected graph. For any collection of orientation coefficients, $\Omega$, there exists a collection of skew-zigzag coefficients, $v$, such that $B_{\Omega}^{\Gamma}=A_{v}(\Gamma)$.

Proof. Let $\Omega=\left(\epsilon_{a, b}\right)$ be a collection of orientation coefficients and set $v_{b, c}^{a}=\epsilon_{a, c} / \epsilon_{a, b}$ for all $\{a, b\},\{a, c\} \in E$. For any $\{a, b\},\{a, c\},\{a, d\} \in E$ we have

$$
v_{b, b}^{a}=\frac{\epsilon_{a, b}}{\epsilon_{a, b}}=1, \quad v_{b, c}^{a} v_{c, b}^{a}=\frac{\epsilon_{a, c}}{\epsilon_{a, b}} \frac{\epsilon_{a, b}}{\epsilon_{a, c}}=1, \quad \text { and } \quad v_{b, c}^{a} v_{c, d}^{a} v_{d, b}^{a}=\frac{\epsilon_{a, c}}{\epsilon_{a, b}} \frac{\epsilon_{a, d}}{\epsilon_{a, c}} \frac{\epsilon_{a, b}}{\epsilon_{a, d}}=1 .
$$

Thus the set $v=\left(v_{b, c}^{a} \mid\{a, b\},\{a, c\} \in E\right)$ is a collection of skew-zigzag coefficients. It is clear that $I_{\Omega}=I_{v}$. Thus, we have $B_{\epsilon}^{\Gamma}=A_{v}(\Gamma)$.

Definition 5.2 (orientation). Let $\Gamma=(V, E)$ be a connected graph and let $D \Gamma=\left(V, E^{\prime}\right)$ be its double graph. A set $\epsilon \subseteq E^{\prime}$ is said to be an orientation of $D \Gamma$ if, for every $\{a, b\} \in E$, exactly one directed edge in $D \Gamma$ between $a$ and $b$ is in $\epsilon$.

In [3, Section 2.1, p. 109] the authors fix an orientation $\epsilon$ of $D \Gamma$. Then, they define orientation coefficients $\Omega=\left(\epsilon_{a, b} \mid a, b \in V\right)$ as follows:

$$
\epsilon_{a, b}= \begin{cases}1 & \text { if }(a \mid b) \in \epsilon \\ -1 & \text { if }(b \mid a) \in \epsilon \\ 0 & \text { if } a \text { and } a \text { are not connected }\end{cases}
$$

Notice that $\epsilon_{a, b}=-\epsilon_{b, a}$. Thus, by Lemma 5.1, we have $B_{\Omega}^{\Gamma}=A_{v}(\Gamma)$, where

$$
v=\left(v_{b, c}^{a}=\frac{\epsilon_{a, c}}{\epsilon_{a, b}} \mid\{a, b\},\{a, c\} \in E\right) .
$$

However, the following proposition shows that the converse of Lemma 5.1 is false. Thus, the alternate definition of skew-zigzag algebras in terms of orientation coefficients is more restrictive.

Proposition 5.3. If $\Gamma$ is not a bipartite graph, then $A(\Gamma)$ is not isomorphic to $B_{\Omega}^{\Gamma}$ for any collection of orientation coefficients $\Omega$.

Proof. Suppose that $\Gamma$ is not a bipartite graph and that there exists an isomorphism

$$
\phi: A(\Gamma) \rightarrow B_{\Omega}^{\Gamma}
$$

for some collection of orientation coefficients $\Omega$. For all $a \in V$ let $\phi([a])=\left[x_{a}\right]$. So, for all $\{a, b\} \in E$ we have $\phi([a \mid b])=\alpha_{a, b}\left[x_{a} \mid x_{b}\right]$ for some $\alpha_{a, b} \in \mathbb{k}$. Consequently, for any $\{a, b\}$, $\{a, c\} \in E$ we have

$$
\alpha_{a, b} \alpha_{b, a}\left[x_{a}\left|x_{b}\right| x_{a}\right]=\phi([a|b| a])=\phi([a|c| a])=\alpha_{a, c} \alpha_{c, a}\left[x_{a}\left|x_{c}\right| x_{a}\right] .
$$

Therefore,

$$
\frac{\alpha_{a, c} \alpha_{c, a}}{\alpha_{a, b} \alpha_{b, a}}=\frac{\epsilon_{x_{a}, x_{c}}}{\epsilon_{x_{a}, x_{b}}} .
$$

By [4, Proposition 1.6.1], since $\Gamma$ is not bipartite, it contains a cycle of odd length. Thus, there is a cycle $C=\left(a_{1}|\ldots| a_{n}\right)$ in $D \Gamma$ with $n$ even. So, (5.2) yields

$$
\begin{aligned}
& 1=\frac{\alpha_{a_{1}, a_{2}} \alpha_{a_{2}, a_{1}}}{\alpha_{a_{1}, a_{n-1}} \alpha_{a_{n-1}, a_{1}}} \frac{\alpha_{a_{2}, a_{3}} \alpha_{a_{3}, a_{2}}}{\alpha_{a_{2}, a_{1}} \alpha_{a_{1}, a_{2}}} \cdots \frac{\alpha_{a_{n-1}, a_{1}} \alpha_{a_{1}, a_{n-1}}}{\alpha_{a_{n-1}, a_{n-2}} \alpha_{a_{n-2}, a_{n-1}}} \\
& =\frac{\epsilon_{x_{a_{1}}, x_{a_{2}}}}{\epsilon_{x_{a_{1}}, x_{a_{n-1}}}} \frac{\epsilon_{x_{a_{2}}, x_{a_{3}}}}{\epsilon_{x_{a_{2}}, x_{a_{1}}}} \cdots \frac{\epsilon_{x_{a_{n-1}}, x_{a_{1}}}}{\epsilon_{x_{a_{n-1}}, x_{a_{n-2}}}} .
\end{aligned}
$$


Since $\left\{a_{i}, a_{i+1}\right\} \in E$ for all $1 \leq i \leq n-1, \epsilon_{x_{a_{j}}, x_{a_{k}}} \neq 0$ for $1 \leq j, k, \leq n-1, j \neq k$. Moreover, we know that $\epsilon_{a, b}=-\epsilon_{b, a}$ for all $a, b \in V$. Consequently, (5.3) yields

$$
1=(-1)^{n-1}=-1 \text {. }
$$

This contradiction implies that $A_{u}(\Gamma) \supsetneqq B_{\Omega}^{\Gamma}$ for any $\Omega$.

Suppose $\left(\epsilon_{a, b}\right)$ is a collection of orientation coefficients. For $x \in V$, define $V_{x}$ and $y_{x}$ as in Proposition 3.7. Notice that if we modify the set $J$ in (3.2) by setting

$$
J^{\prime}:=\left\{[a],[b \mid c], \epsilon_{x, y_{x}}\left[x\left|y_{x}\right| x\right] \mid a, x \in V, b, c \in V \text { such that }\{b, c\} \in E\right\},
$$

then $J^{\prime}$ is independent of the choice of $y_{x}$ for every $x \in V$. Indeed, for any $y, z \in V_{x}$, we have

$$
\epsilon_{x, y}[x|y| x]=\epsilon_{x, z}[x|z| x] .
$$

In $\left[2\right.$, Section 6.1, p. 2516], the authors fix an orientation $\epsilon$ of $\Gamma$ and define coefficients $\epsilon_{a, b}$ $(a, b \in V)$ as in (5.1). They then define a diagrammatic algebra using these coefficients. Their algebra is in fact isomorphic to the algebra $B_{\Omega}^{\Gamma}$, for $\Omega=\left(\epsilon_{a, b} \mid a, b \in V\right)$, via the map

$$
A \rightarrow A(\Gamma), \quad \oint_{x}^{y} \mapsto\left\{\begin{array}{ll}
{[x \mid y]} & x \neq y, \\
\epsilon_{x, y_{x}}\left[x\left|y_{x}\right| x\right] & \text { if } x=y,
\end{array} \quad x \mapsto[x] .\right.
$$

So, by Lemma 5.1, $A$ is isomorphic to $A_{v}(\Gamma)$ where $v=\left(v_{b, c}^{a}=\frac{\epsilon_{a, c}}{\epsilon_{a, b}} \mid\{a, b\},\{a, c\} \in E\right)$. However, if $\Gamma$ is not bipartite, then this is not isomorphic to the zigzag algebra by Proposition 5.3.

Note on the $\mathbf{H}_{\mathbf{A}} \mathbf{T} \mathbf{X}$ version. For the interested reader, the tex file of this paper includes hidden details of some straightforward computations and arguments that are omitted in the pdf file. These details can be displayed by switching the details toggle to true in the tex file and recompiling.

\section{Acknowledgements}

This work was completed under the supervision of Professor Alistair Savage. The author would like to thank Professor Savage immensely for his patience and guidance throughout this paper as well as the opportunity to write this paper. The author would also like to thank the University of Ottawa and the Work-Study Program for their support. Finally, the author would like to thank the referees for their useful comments and for providing a reference for Proposition 3.9.

\section{References}

[1] Assem I., Simson D., Skowroński A., Elements of the representation theory of associative algebras. Vol. 1. Techniques of representation theory, London Mathematical Society Student Texts, Vol. 65, Cambridge University Press, Cambridge, 2006.

[2] Cautis S., Licata A., Heisenberg categorification and Hilbert schemes, Duke Math. J. 161 (2012), 2469-2547, arXiv:1009.5147.

[3] Cautis S., Licata A., Sussan J., Braid group actions via categorified Heisenberg complexes, Compos. Math. 150 (2014), 105-142, arXiv:1207.5245.

[4] Diestel R., Graph theory, Graduate Texts in Mathematics, Vol. 173, 4th ed., Springer, Heidelberg, 2010, available at http://diestel-graph-theory.com/. 
[5] Huerfano R.S., Khovanov M., A category for the adjoint representation, J. Algebra 246 (2001), 514-542, math.QA/0002060.

[6] Kock J., Frobenius algebras and 2D topological quantum field theories, London Mathematical Society Student Texts, Vol. 59, Cambridge University Press, Cambridge, 2004.

[7] Rosso D., Savage A., A general approach to Heisenberg categorification via wreath product algebras, arXiv:1507.06298.

[8] Sunada T., Topological crystallography. With a view towards discrete geometric analysis, Surveys and Tutorials in the Applied Mathematical Sciences, Vol. 6, Springer, Tokyo, 2013.

[9] Zimmermann A., Representation theory. A homological algebra point of view, Algebra and Applications, Vol. 19, Springer, Cham, 2014. 